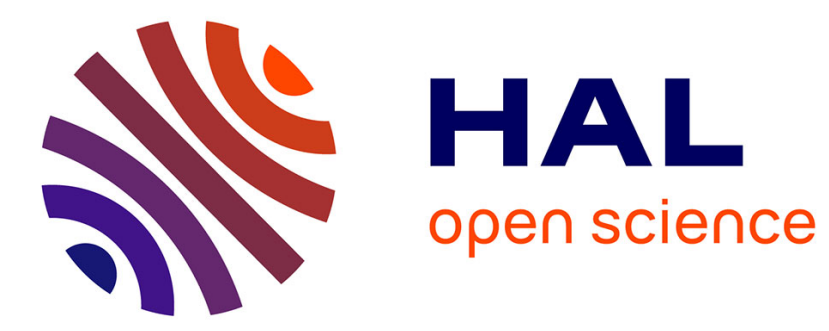

\title{
Homogenization of acoustic metamaterials using retrieval methods
}

Laetitia Roux, Charles Croënne, Christian Audoly, Anne-Christine Hladky

\section{To cite this version:}

Laetitia Roux, Charles Croënne, Christian Audoly, Anne-Christine Hladky. Homogenization of acoustic metamaterials using retrieval methods. Journal of Applied Physics, 2020, 127 (22), pp.225102. 10.1063/5.0002859 . hal-03019987

\section{HAL Id: hal-03019987 \\ https://hal.science/hal-03019987}

Submitted on 23 Nov 2020

HAL is a multi-disciplinary open access archive for the deposit and dissemination of scientific research documents, whether they are published or not. The documents may come from teaching and research institutions in France or abroad, or from public or private research centers.
L'archive ouverte pluridisciplinaire HAL, est destinée au dépôt et à la diffusion de documents scientifiques de niveau recherche, publiés ou non, émanant des établissements d'enseignement et de recherche français ou étrangers, des laboratoires publics ou privés. 


\section{Homogenization of acoustic metamaterials using retrieval methods}

Cite as: J. Appl. Phys. 127, 225102 (2020); https://doi.org/10.1063/5.0002859

Submitted: 29 January 2020 . Accepted: 25 May 2020. Published Online: 09 June 2020

Laetitia Roux, Charles Croënne, Christian Audoly, and (D) Anne-Christine Hladky-Hennion

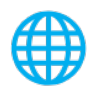

\section{ARTICLES YOU MAY BE INTERESTED IN}

Multistable metamaterial on elastic foundation enables tunable morphology for elastic wave control

Journal of Applied Physics 127, 225104 (2020); https://doi.org/10.1063/1.5145324

Gradient-index phononic crystals for omnidirectional acoustic wave focusing and energy harvesting

Applied Physics Letters 116, 234101 (2020); https://doi.org/10.1063/5.0008791

Broadband integrative acoustic asymmetric focusing lens based on mode-conversion metaatoms

Applied Physics Letters 116, 223505 (2020); https://doi.org/10.1063/5.0004579

New

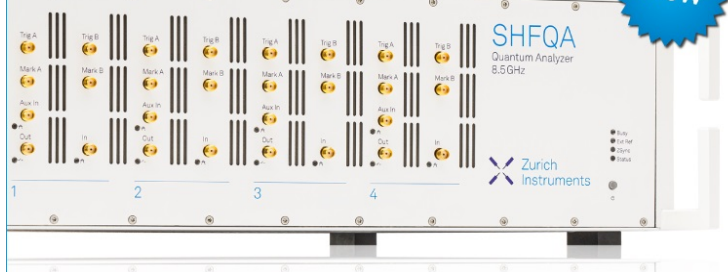

Your Qubits. Measured.

Meet the next generation of quantum analyzers

- Readout for up to 64 qubits

- Operation at up to $8.5 \mathrm{GHz}$, mixer-calibration-free

- Signal optimization with minimal latency
Find out more

Zurich

Instruments 


\title{
Homogenization of acoustic metamaterials using retrieval methods
}

Cite as: J. Appl. Phys. 127, 225102 (2020); doi: 10.1063/5.0002859

Submitted: 29 January 2020 . Accepted: 25 May 2020 .

Published Online: 9 June 2020

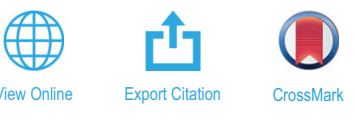

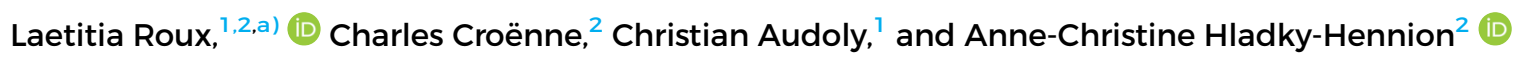

\author{
AFFILIATIONS \\ ${ }^{7}$ Naval Group Research, 831900 Ollioules, France \\ ${ }^{2}$ CNRS, Centrale Lille, ISEN, Univ. Lille, Univ. Valenciennes, UMR 8520-IEMN, 59000 Lille, France
}

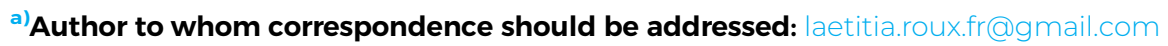

\begin{abstract}
The effective properties of two-dimensional acoustic metamaterials are here obtained by applying two retrieval methods, referred to as the direct inversion method and the differential method. They employ the scattering coefficients at the incidence and transmission sides of the global systems immersed in a fluid. A validation case study on a one-dimensional periodic design is first presented. A two-dimensional design comprising periodic cylindrical steel inclusions in a soft elastic matrix is then examined. Homogenization issues related to different underlying assumptions in the two retrieval methods are discussed. It is shown that one of the retrieval methods (the differential method) well describes the interior of the medium, away from the interfaces, while the other (the direct inversion method) captures the interface effects. The two retrieval methods are used to build homogeneous equivalent media which are used to predict scattering coefficients. A hybrid method is introduced which combines the two methods to create effective media that are accurate approximations of their $2 \mathrm{D}$ counterparts.
\end{abstract}

Published under license by AIP Publishing. https://doi.org/10.1063/5.0002859

\section{INTRODUCTION}

Metamaterials are artificial materials with the capacity to exhibit unusual properties arising from the collective manifestation of the internal constituent units in the structure. ${ }^{1}$ This concept has offered new perspectives for the design of noise control materials. Utilizing their ability to manipulate wave propagation, metamaterials have been employed in a wide range of applications including lensing, ${ }^{2}$ sound focusing, ${ }^{3}$ switching, ${ }^{4}$ and cloaking. ${ }^{5}$ Their study commonly includes the determination of complex effective properties of an equivalent homogeneous medium, often a fluid. These effective properties can strongly vary with frequency and reach negative values for frequencies associated with local resonances of the inclusions. ${ }^{6-8}$ In acoustic metamaterials, different types of inclusions have been proposed to achieve negative density and/or negative bulk modulus. Monopole resonance of voids in a soft elastic medium has been shown to lead to negative effective bulk modulus, ${ }^{9}$ whereas dipole resonance of hard scatterers in an elastic medium has been shown to lead to negative effective density. ${ }^{10,11} \mathrm{~A}$ combination of monopole and dipole scatterers has been demonstrated to achieve simultaneous negative density and bulk modulus for broadband acoustic performance. ${ }^{12,13}$
The effective wavenumber is commonly calculated to identify frequency ranges where no wave propagation occurs. It is also often assumed to be identical to the Bloch wavenumber of an infinite periodic medium. A range of analytical techniques has been employed to derive this effective wavenumber in one-dimensional (1D) designs comprising successive material layers ${ }^{14,15}$ and in twodimensional (2D) designs comprising periodically voided soft elastic media. ${ }^{16,17}$ While analytical methods provide insight into physical mechanisms for wave propagation and are generally computationally efficient, they are limited to simple geometric designs. Wave propagation in periodic systems can be conveniently simulated using element-based numerical methods. In early work based on the finite element method (FEM), Hladky-Hennion and Decarpigny ${ }^{16}$ developed a code to compute sound transmission in a rubber medium submerged in water and embedded with periodic cylindrical cavities of finite height. A unit cell of the coating comprising a single cavity in rubber was simulated and periodicity of the geometry was implemented by applying classical Bloch conditions on the boundaries of the unit cell. Using the same finite element code, Langlet et al. ${ }^{18}$ calculated the effective wavenumber of a periodically voided elastic medium. More recently, FEM has 
been employed to investigate material dissipative effects on wave dispersion and bandgaps in acoustic metamaterial designs. ${ }^{19,20}$

The calculation of dispersion curves for periodic systems does not take into account interface effects and eventual coupling with a surrounding medium. Retrieval methods are an alternative approach to derive effective wavenumber as well as other effective properties using scattering coefficients obtained on the incidence and transmission sides of the medium. ${ }^{21,22}$ For such methods, the scattering coefficients have been obtained analytically for a periodic multilayered medium, ${ }^{23,24}$ numerically for periodic metamaterials, ${ }^{25,26}$ or experimentally for acoustic metamaterials composed of split hollow spheres ${ }^{27}$ and periodically arranged hollow tubes. ${ }^{28}$ Park et al. also proposed a method to homogenize anisotropic metamaterial slabs by determining effective properties of the constitutive unit cell. ${ }^{29}$ Within the slab, the effective properties differ whether the unit cell is an edge cell or an inner cell.

The emphasis here is on the homogenization of metamaterials for underwater applications. A metamaterial comprising hard inclusions embedded in a viscoelastic matrix is an ideal candidate as an external coating on the hull of an underwater vehicle, attributed to impedance matching of the viscoelastic medium with water, to the presence of local resonances of the inclusions, ${ }^{11,13,30}$ and to the robustness of the hard scatterers under hydrostatic pressure. ${ }^{26}$ Scattering of sound waves by resonant inclusions results in the conversion of longitudinal waves into shear waves, the latter being easily dissipated in a viscoelastic medium. ${ }^{31}$ Performance of such systems can be improved by coating hard inclusions with a viscous rubber layer ${ }^{32}$ or by using multiple layers of scatterers of different resonant frequencies to broaden the acoustic performance. ${ }^{33}$ It is, therefore, interesting to homogenize several of these designs in order to use their effective properties in a topological optimization process.

Our leading goal is to develop a computationally efficient tool to optimize complex two-dimensional metamaterials. To achieve this, it is beneficial to effectively replace any $2 \mathrm{D}$-medium, which may comprise inclusions of various shapes and sizes, by a medium made of successive layers of fluid. An effective medium approach is, therefore, presented in Sec. II. In this work, two retrieval methods are employed to determine the effective properties of a constituent unit cell for different $1 \mathrm{D}$ and $2 \mathrm{D}$ periodic designs, considering only normally incident plane waves in water. Both retrieval methods are based on a fluid homogenization model, whereby the effective equivalent fluid properties of a unit cell are characterized by an effective wavenumber and an effective impedance. Both methods also require a priori knowledge of the scattering coefficients on the incidence and transmission sides of the global system. In the direct inversion method, the global transfer matrix in terms of the scattering coefficients is equated with the product of the transfer matrices for each periodic cell. In the differential method, the transfer matrix of a periodic cell in the core of the medium is derived from the product of the global transfer matrices of two periodic media differing only by length. In Sec. III, two case studies are examined, starting with a $1 \mathrm{D}$ design comprising alternating layers of soft and rigid media for which results obtained from a model based on the transfer matrix method are compared with results from the proposed retrieval approaches. A 2D design comprising periodic cylindrical rigid inclusions in a soft elastic matrix is then considered. Effective parameters of a unit cell are used to predict the scattering responses for various numbers of unit cells. A hybrid prediction method is introduced which combines the two retrieval methods similar to the work by Park et al.; ${ }^{29}$ we herein distinguish effective properties for inner or edge cells. Our work extends the prediction capability as effective material parameters are retrieved well beyond the low frequency. We also demonstrate the ability to homogenize complex systems considering a heavy fluid environment (water), with fluid-structure interactions always involving shear waves and with the presence of strong resonances that generates significant near-field effects. Homogenization of the metamaterials under study is, therefore, challenging but the prediction tool developed here can work effectively for the homogenization and optimization of 2D metamaterials since it helps building homogeneous equivalent media which are a precise approximation of their $2 \mathrm{D}$ counterparts.

\section{METHODOLOGY}

\section{A. Fluid homogenization model}

A fluid homogenization model is employed to characterize the effective medium in terms of a complex effective wavenumber and effective impedance. We herein consider a medium immersed in water and subject to an acoustic harmonic plane wave of unity amplitude at normal incidence given by $e^{\mathrm{i}(\omega t-k x)}$, where $\mathrm{i}=\sqrt{-1}, \omega$ is the angular frequency, $k$ is the longitudinal wavenumber, $t$ denotes time, and $x$ defines the position on the longitudinal axis. A transfer matrix approach is adopted that relates the pressure and normal particle velocity on the incidence and transmission sides of the medium as follows: ${ }^{34}$

$$
\left(\begin{array}{c}
p_{\text {in }} \\
v_{\text {in }}
\end{array}\right)=\mathbf{M}\left(\begin{array}{l}
p_{\text {out }} \\
v_{\text {out }}
\end{array}\right),
$$

where $p_{\text {in }}$ and $p_{\text {out }}$ are, respectively, the pressure at the inlet and outlet of the medium, and similarly, $v_{\text {in }}$ and $v_{\text {out }}$ are, respectively, the normal particle velocity at the medium inlet and outlet.

\section{B. Direct inversion method}

Figure 1 presents a segmented system comprising periodic repetitions of a constituent unit cell, each of length $L_{u} . R$ and $T$, respectively, correspond to the coefficients of the reflected and transmitted pressures on the incidence and transmission sides of the global medium. All unit cells are identical.

On the one hand, the global transfer matrix $\mathbf{M}_{n}$ of the periodic medium comprising $n$ unit cells can be calculated using its

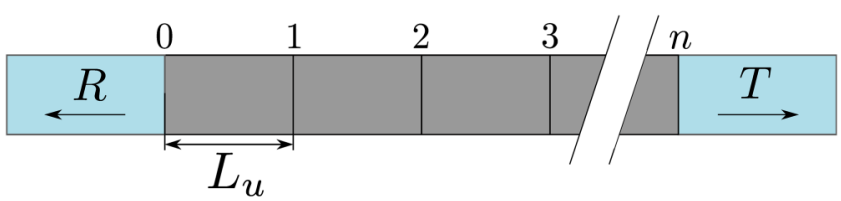

FIG. 1. Schematic diagram of a segmented medium comprising $n$ identical unit cells. Reflection and transmission coefficients on the incidence and transmission sides of the medium are also shown. 
scattering coefficients as follows: ${ }^{35,36}$

$$
\mathbf{M}_{n}=\frac{1}{2 T}\left[\begin{array}{cc}
1-R^{2}+T^{2} & \left((1+R)^{2}-T^{2}\right) Z_{f} \\
\left((1-R)^{2}-T^{2}\right) Z_{f}^{-1} & 1-R^{2}+T^{2}
\end{array}\right] .
$$
by ${ }^{34}$

On the other hand, the transfer matrix for each cell is given

$$
\mathbf{M}_{u}=\left[\begin{array}{cc}
\cos \left(k_{\mathrm{dir}} L_{u}\right) & \mathrm{i} Z_{\mathrm{dir}} \sin \left(k_{\mathrm{dir}} L_{u}\right) \\
\mathrm{i} Z_{\mathrm{dir}}^{-1} \sin \left(k_{\mathrm{dir}} L_{u}\right) & \cos \left(k_{\mathrm{dir}} L_{u}\right)
\end{array}\right]
$$

where $k_{\text {dir }}$ is the effective longitudinal wavenumber, $Z_{\text {dir }}$ is the effective impedance of the unit cell, and $Z_{f}$ is the impedance of the surrounding fluid on the incidence and transmission sides of the global medium. The global transfer matrix of the segmented medium can also be written as the product of the transfer matrix for each cell, which leads to $\mathbf{M}_{n}=\left(\mathbf{M}_{u}\right)^{n}$. The calculation of $\left(\mathbf{M}_{u}\right)^{n}$ for any integer $n$ is equivalent to replacing only the thickness $L_{u}$ by $n L_{u}$. The global transfer matrix of the system then becomes

$$
\left(\mathbf{M}_{u}\right)^{n}=\left[\begin{array}{cc}
\cos \left(n k_{\mathrm{dir}} L_{u}\right) & \mathrm{i} Z_{\mathrm{dir}} \sin \left(n k_{\mathrm{dir}} L_{u}\right) \\
\mathrm{i} Z_{\mathrm{dir}}^{-1} \sin \left(n k_{\mathrm{dir}} L_{u}\right) & \cos \left(n k_{\mathrm{dir}} L_{u}\right)
\end{array}\right] .
$$

The effective impedance is, thus, given by

$$
Z_{\text {dir }}= \pm \sqrt{\frac{\mathbf{M}_{n_{1,2}}}{\mathbf{M}_{n_{2,1}}}}
$$

where $\mathbf{M}_{n_{i, j}}(1 \leq i, j \leq 2)$ denote the elements of the global transfer matrix $\mathbf{M}_{n}$. For the time convention $e^{\mathrm{i} \omega t}$ adopted in the current work, passivity constraints set the sign of the real part of the effective impedance to be positive. ${ }^{37}$ The effective wavenumber is then obtained using either $\mathbf{M}_{n_{1,1}}$ or $\mathbf{M}_{n_{2,2}}$ as follows:

$$
k_{\mathrm{dir}}= \pm \frac{\cos ^{-1}\left(\mathbf{M}_{n_{1,1}}\right)}{n L_{u}}+\frac{2 \pi m}{n L_{u}} .
$$

The passive material condition is satisfied when the imaginary part of $k_{\text {dir }}$ is negative. Integer $m$ is chosen to ensure continuity of the real part of $k_{\text {dir }}$ as a function of frequency. It is worth noting that this phase continuity constraint is largely applied in literature studies. ${ }^{22-24,27,29}$ It ensures that the group velocity and the acoustic energy flux can be defined for the homogenized medium at all frequencies.

\section{Differential method}

The second retrieval method presented here is an extension of the Bianco and Parodi method, ${ }^{21}$ in which two media are considered, differing only in length by $\Delta L$ in the direction of wave propagation. The propagation constant in a medium of infinite length is obtained by examining wave propagation within the section $\Delta L$ of the longer finite medium.

A particular case is herein considered, where the two media are modeled as segmented media comprising periodic repetitions of a constituent unit cell, as shown in Fig. 2. The extra portion in the longer medium is a unit cell of length $L_{u}$. The shorter segmented medium comprises $n$ unit cells and the longer medium comprises $n+1$ unit cells. $R_{i}$ and $T_{i}(i=1,2)$, respectively, correspond to the coefficients of the reflected and transmitted pressures calculated at the interfaces between the two media and the surrounding fluid.

The global transfer matrix of the shorter periodic medium comprising $n$ unit cells can be expressed in terms of its scattering coefficients as follows:

$$
\mathbf{M}_{n}=\frac{1}{2 T_{1}}\left[\begin{array}{cc}
1-R_{1}^{2}+T_{1}^{2} & \left(\left(1+R_{1}\right)^{2}-T_{1}^{2}\right) Z_{f} \\
\left(\left(1-R_{1}\right)^{2}-T_{1}^{2}\right) Z_{f}^{-1} & 1-R_{1}^{2}+T_{1}^{2}
\end{array}\right] .
$$

Similarly, the transfer matrix of the longer segmented medium is given by

$$
\mathbf{M}_{n+1}=\frac{1}{2 T_{2}}\left[\begin{array}{cc}
1-R_{2}^{2}+T_{2}^{2} & \left(\left(1+R_{2}\right)^{2}-T_{2}^{2}\right) Z_{f} \\
\left(\left(1-R_{2}\right)^{2}-T_{2}^{2}\right) Z_{f}^{-1} & 1-R_{2}^{2}+T_{2}^{2}
\end{array}\right] .
$$

The shorter medium is virtually segmented in two sections referred to as section $1\left(s_{1}\right)$ and section $2\left(s_{2}\right)$, for which the transfer matrices are, respectively, $\mathbf{M}_{\mathrm{s}_{1}}$ and $\mathbf{M}_{\mathrm{s}_{2}}$. The global transfer matrix for the shorter medium is then given by $\mathbf{M}_{n}=\mathbf{M}_{\mathrm{s}_{1}} \mathbf{M}_{\mathrm{s}_{2}}$. The global transfer matrix for the longer medium requires inclusion of the transfer matrix associated with $L_{u}$ and is given by $\mathbf{M}_{n+1}=\mathbf{M}_{\mathrm{s}_{1}} \mathbf{M}_{u} \mathbf{M}_{\mathrm{s}_{2}}$. As similar matrices have the same trace, multiplying $\mathbf{M}_{n+1}$ by the inverse of $\mathbf{M}_{n}$ yields the following trace equality:

$$
\operatorname{Tr}\left(\mathbf{M}_{n+1}\left(\mathbf{M}_{n}\right)^{-1}\right)=\operatorname{Tr}\left(\mathbf{M}_{u}\right)
$$

Assuming that wave propagation within the second medium extra portion $L_{u}$ is monomode and described by the effective wavenumber $k_{\text {diff }}$, the fluid homogenization model is applied (only) to this core portion $L_{u}$ which is then described by a transfer matrix under the form given by Eq. (3), yielding $\operatorname{Tr}\left(\mathbf{M}_{u}\right)=2 \cos \left(k_{\text {diff }} L_{u}\right)$.

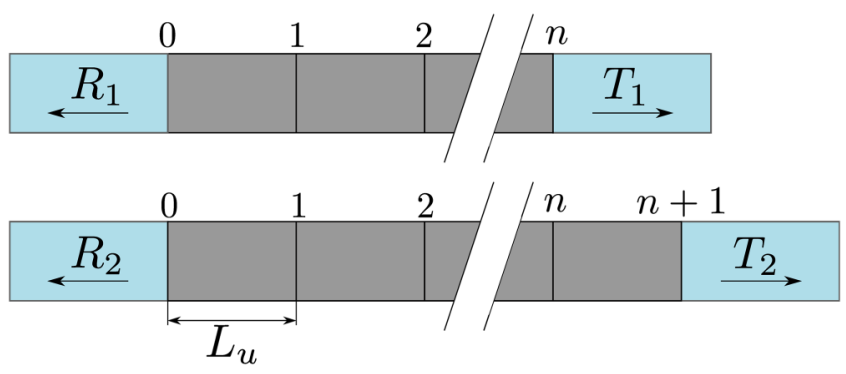

FIG. 2. Schematic diagram illustrating the differential method in which two finite segmented media comprising identical unit cells differ only by a single unit cell. Reflection and transmission coefficients of the two segmented media are also shown. 
The expression for the effective wavenumber can be obtained as

$$
k_{\text {diff }}= \pm \frac{\cos ^{-1}\left(\frac{\operatorname{Tr}\left(\mathbf{M}_{n+1}\left(\mathbf{M}_{n}\right)^{-1}\right)}{2}\right)}{L_{u}}+\frac{2 \pi m}{L_{u}}
$$

where $m$ is an integer whose value is determined such that the effective wavenumber as a function of frequency is continuous. It should be noted that in contrast to Eq. (6), the effective wavenumber given by Eq. (10) corresponds to the wavenumber in the medium core. ${ }^{25}$

The effective impedance is now derived. Equation (5), for the direct inversion method, has been derived using the theoretical expressions for the elements of the transfer matrix as given in Eq. (3), which are valid to describe a fluid. In the differential method, the effective medium is also assumed to be a fluid but the theoretical expressions of the transfer matrix elements are not directly used to derive the effective impedance. Instead, the transfer matrices of the segmented media are written as the product of the transfer matrix for each unit cell, yielding $\mathbf{M}_{n}=\left(\mathbf{M}_{u}\right)^{n}$ for the shorter medium and $\mathbf{M}_{n+1}=\left(\mathbf{M}_{u}\right)^{n+1}$ for the longer medium. The transfer matrix $\mathbf{M}_{u}$ of a unit cell can now be obtained from the product of the global transfer matrix of the longer segmented medium with the inverse of that of the shorter medium, that is,

$$
\mathbf{M}_{n+1}\left(\mathbf{M}_{n}\right)^{-1}=\left(\mathbf{M}_{u}\right)^{n+1-n}=\mathbf{M}_{u} .
$$

Using Eqs. (7) and (8), the left hand side of Eq. (11) can be calculated in terms of the scattering responses of the two segmented media. Using Eq. (1) and the relation $Z_{\text {eff }}=p_{\text {in }} / v_{\text {in }}=p_{\text {out }} / v_{\text {out }}$ for fluid homogenization, the effective impedance can be obtained as

$$
Z_{\text {diff }}=\frac{-\left(\mathbf{M}_{u_{2,2}}-\mathbf{M}_{u_{1,1}}\right) \pm \sqrt{\left(\mathbf{M}_{u_{2,2}}-\mathbf{M}_{u_{1,1}}\right)^{2}+4 \mathbf{M}_{u_{1,2}} \mathbf{M}_{u_{2,1}}}}{2 \mathbf{M}_{u_{2,1}}}
$$

where $\mathbf{M}_{u_{i, j}}(1 \leq i, j \leq 2)$ corresponds to the elements of the transfer matrix $\mathbf{M}_{u}$ given by Eq. (11). The approach to derive Eq. (12) is, therefore, different than that of the direct inversion method. The reason is that the fluid homogenization model may be too simplistic and may fail to describe complex systems exhibiting shear wave propagation, coupling with a surrounding heavy fluid and so on. For such cases, we observed that the calculation of the transfer matrix of the unit cell as per Eq. (11) does not always lead to a transfer matrix in which the diagonal terms are identical as they are meant to be according to Eq. (3). Such cases can nonetheless be considered when using Eq. (12).

\section{Scattering coefficients}

The scattering coefficients are numerically calculated using the FEM code ATILA. ${ }^{38}$ A small domain of water is modeled on the incidence and transmission sides of the media, with the fluid speed of sound set as $c_{f}=1500 \mathrm{~m} / \mathrm{s}$ and fluid density $\rho_{f}=1000 \mathrm{~kg} / \mathrm{m}^{3}$. The effects of the remaining fluid domain are accounted for by matching the pressure field in the finite element mesh with simple plane wave expansions of the in-going and out-going waves, similarly to the work of Hladky-Hennion and Decarpigny. ${ }^{16}$ Each periodic medium is subject to harmonic plane wave excitation at normal incidence from the fluid domain. A periodic boundary condition is applied on the lateral boundaries of the medium to simulate an infinite array of unit cells in the direction transverse to the direction of sound propagation. Reflection and transmission coefficients are calculated from the pressure at the interface between the water and the incidence and transmission sides of the media, respectively.

It has to be noted that in practice, for two-dimensional media, the scattering coefficients are not directly calculated at the interfaces but are taken further away and then phase-shifted to be brought back at the interfaces. This procedure effectively ensures that, in the presence of a non-uniform near field, the scattering coefficients describe the behavior of the finite structure in the far field. It should be pointed out that by definition, the fluid homogenization model used here cannot describe any complex near field at the medium inlet/outlet.

\section{RESULTS AND DISCUSSION}

\section{A. 1D multilayered design}

The effective properties for a 1D multilayered design obtained using the two retrieval methods are initially compared with those obtained using an analytical model. The unit cell of the multilayered design comprises a layer of silicone rubber of thickness $d_{s}$, followed by a layer of aluminum of thickness $d_{\mathrm{a}}$, and then another silicone rubber of thickness $d_{s}$, as shown in Fig. 3(a). The density of silicone and aluminum are, respectively, $\rho_{\mathrm{s}}=1250 \mathrm{~kg} \mathrm{~m}^{-3}$ and $\rho_{\mathrm{a}}=2700 \mathrm{~kg} \mathrm{~m}^{-3}$. The longitudinal speed of sound for silicone and aluminum are set as $1000 \times(1+0.02 \mathrm{i}) \mathrm{m} \mathrm{s}^{-1}$ and $6200 \mathrm{~m} \mathrm{~s}^{-1}$, respectively. The thickness of silicone and aluminum are selected as $d_{\mathrm{s}}=15 \mathrm{~mm}$ and $d_{\mathrm{a}}=10 \mathrm{~mm}$, hence the length of a unit cell becomes $L_{u}=40 \mathrm{~mm}$. The direct inversion method is applied to the multilayered medium comprising $n=4$ unit cells, as shown in Fig. 3(b). The differential method is applied for $(n, n+1)=(4,5)$.

The model used to validate the retrieval method for this multilayered medium is based on the fact that a symmetric multilayered unit can effectively be replaced by a fluid medium of wavenumber $k_{\text {cell }}$ and impedance $Z_{\text {cell }}$. The transfer matrix of the unit cell is numerically calculated by multiplying the transfer matrices for each

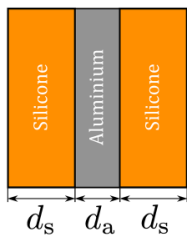

(a)

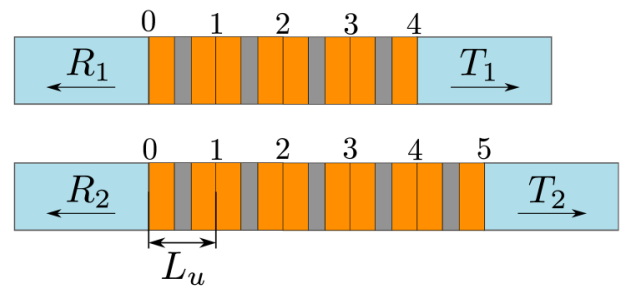

(b)
FIG. 3. (a) Representation of the symmetric multilayered unit cell comprising layers of silicone and aluminum. (b) Multilayered media comprising four and five unit cells for the application of the differential method. The direct inversion method is applied to the medium comprising four unit cells. 
layer within the unit cell using the wavenumber and impedance of silicone rubber and aluminum. Effective parameters can then be extracted from the transfer matrix of the effective unit cell given by

$$
\mathbf{M}_{\text {cell }}=\left[\begin{array}{cc}
\cos \left(k_{\text {cell }} L_{u}\right) & \mathrm{i} Z_{\text {cell }} \sin \left(k_{\text {cell }} L_{u}\right) \\
\mathrm{i} Z_{\text {cell }}^{-1} \sin \left(k_{\text {cell }} L_{u}\right) & \cos \left(k_{\text {cell }} L_{u}\right)
\end{array}\right] .
$$

The transfer matrix based model for periodic multilayered media, also referred to as the symmetric equivalent layer, has be shown to provide an exact representation of periodic and symmetric multilayered unit cells. ${ }^{15}$

Figure 4 presents the dispersion curves in terms of the reduced wavenumber for a frequency range up to $40 \mathrm{kHz}$ obtained by multiplying the transfer matrices of the layers inside a single unit cell ("cell" parameters) and by using the two retrieval methods. The effective wavenumbers using either retrieval methods are in exact agreement with the one derived with the unit cell calculation. The same observation is made for the effective impedance, not shown here.

Assuming zero damping, distinct frequency ranges corresponding to bandgaps occur where the real part of the effective reduced wavenumber is equal to 0 or $\pi$, represented by the shaded areas. This is attributed to the fact that at the interface between the layers of aluminum and silicone, an incoming wave is transferred into a reflected wave which constructively interferes with the incoming wave. The imaginary part of the wavenumber (which represents the wave evanescence) reaches a local maximum, corresponding to greater evanescence of acoustic waves in the bandgaps than in the passbands. Furthermore, the local maxima increase with frequency, resulting in greater attenuation of the acoustic waves with increasing frequency. Consequently, the homogenized units obtained with the retrieval methods are accurate equivalents of the multilayered system and accurately capture evanescence of acoustic waves within bandgaps.

\section{B. 2D hard scatterers}

In $2 \mathrm{D}$ systems, additional physical phenomena such as local resonances and boundary effects occur compared to $1 \mathrm{D}$ periodic systems. It, therefore, becomes more difficult to apply a simple homogenization model, especially for metamaterials with complex geometry. Several homogenization difficulties will be identified for the case study of a locally resonant unit cell comprising a cylindrical steel inclusion of diameter $d=4 \mathrm{~mm}$ in a square polyurethane matrix with a side length of $L_{u}=10 \mathrm{~mm}$, as shown in Fig. 5(a). The material properties of steel are density of $7800 \mathrm{~kg} \mathrm{~m}^{-3}$, Young's modulus of $215 \mathrm{GPa}$, and Poisson's ratio of 0.31 . The density and speed of sound for longitudinal and shear waves of polyurethane are set as $1100 \mathrm{~kg} \mathrm{~m}^{-3}, 1513 \mathrm{~m} \mathrm{~s}^{-1}$, and $157 \mathrm{~m} \mathrm{~s}^{-1}$, respectively, with a loss factor of $6 \%$ for the speed of sound of shear waves.

Dispersion curves in terms of dimensionless wavenumber are plotted in Fig. 6. It can be observed that both retrieval methods lead to the same effective wavenumber up to approximately $10 \mathrm{kHz}$. Differences appear for frequencies around points A, B, and C. These frequencies correspond to resonant modes for which the displacement fields are given in Fig. 7. Figure 7(A) presents the displacement field in the four-unit medium for frequency A $(13.6 \mathrm{kHz})$. The displacement field exhibits a mass-spring (dipole) resonance where the inclusion represents the rigid mass, translating without undergoing any shape changes, while the host matrix represents the spring that retains the oscillations of the masses. For point $\mathrm{C}(25.7 \mathrm{kHz})$, Fig. $7(\mathrm{C})$ shows that the rubber matrix is moving, whereas the steel inclusions are mostly stationary. The waves scattered by the rigid cylinders are trapped in-between the
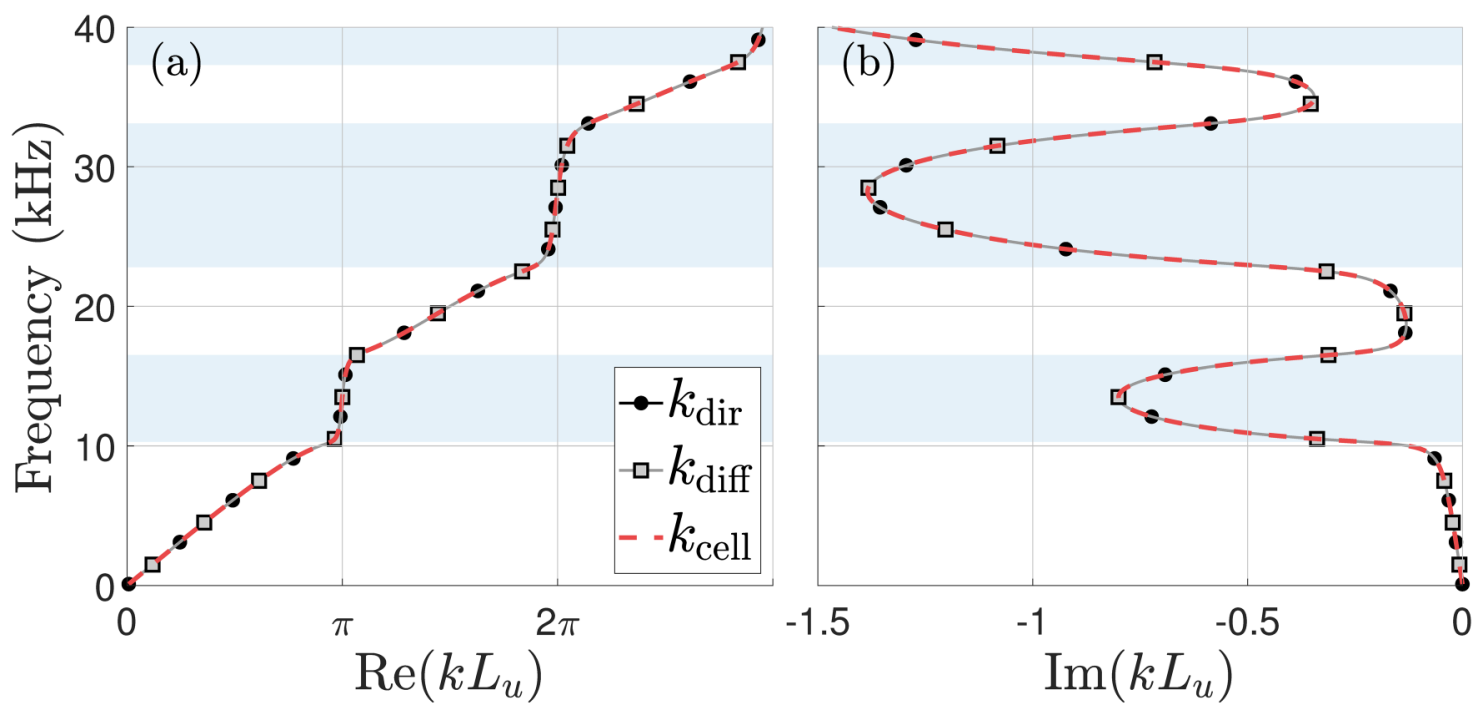

FIG. 4. Dispersion curves for (a) real part and (b) imaginary part of the reduced wavenumber obtained using the direct inversion method $\left(k_{\text {dir }}\right)$, the differential method $\left(k_{\text {diff }}\right)$, and the transfer matrix based model for symmetric the multilayered unit $\left(k_{\text {cell }}\right)$. 


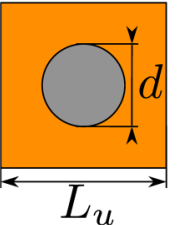

(a)
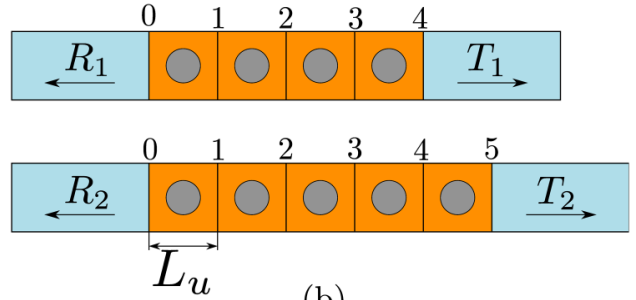

(b)
FIG. 5. (a) Representation of the locally resonant unit cell. (b) Two segmented media comprising $n=4$ and $n+1=5$ unit cells, each cell of length $L_{u}$.

cylinders, leading to resonance of the host rubber. This mode is, therefore, only existing for the matrix portions which are not at the interfaces in contact with the surrounding water. In Fig. 6, as the imaginary part of the wavenumber represents the attenuation per distance, the wavenumber derived with the differential method for resonances $\mathrm{A}$ and $\mathrm{C}$ leads to slightly greater attenuation than the one from the direct inversion method, but the variations remain similar.

Another specific displacement field of the rubber can be observed in Fig. 7(B) for the frequency B $(19.8 \mathrm{kHz})$, but very localized at the input fluid/medium interface. Point B thus also corresponds to a resonant mode in the matrix which only exists between the interface and the first layer of rigid inclusions. As such, unlike the two other resonances, the boundary interference effects of point $B$ do not vary with the number of unit cells placed after the first unit cell at the interface. In Fig. 6, the interface mode at point $\mathrm{B}$ is associated with an attenuation peak for the direct inversion method. Such attenuation is not apparent using the differential method, which aims to describe the interior of the medium away from the boundary interfaces with the fluid. In contrast, the direct inversion method describes the finite medium including boundary interface effects.

The effective density given by $\rho=Z k / \omega$ is presented in Fig. 8, showing slight differences between the two retrieval methods. Nonetheless, both methods show the real part of effective density becoming negative for the mass-spring resonance at point A. This phenomenon has been widely reported in literature studies, ${ }^{1,10,12}$ and is associated with dipole resonance as described previously. The frequency of the dipole resonance has additionally been predicted using an analytical model. ${ }^{11}$

The effective properties for the locally resonant medium differ depending on the retrieval method used, which was not the case for the multilayered medium. This is due to the fact that the fluid homogenization model does not take into consideration complex near-field effects and shear wave propagation, as the effective medium is assumed as a fluid medium in which only longitudinal waves can propagate. This assumption is valid for $1 \mathrm{D}$ systems under normal incidence where only longitudinal modes were excited. However, for $2 \mathrm{D}$ systems made of a solid matrix comprising inclusions, the conversion of longitudinal waves into shear waves due to local resonances cannot be described by the present homogenization model. However, both retrieval methods capture the behavior of the $2 \mathrm{D}$ locally resonant metamaterial with reasonable accuracy. The differential method may be more suitable for most scattering coefficient predictions since, unlike the direct inversion method, the effective parameters are almost independent of the number of unit cells. This can be observed in Fig. 9 which presents the imaginary part of the effective wavenumbers, $k_{\operatorname{dir}_{n}}$ and $k_{\text {diff }}(n, 1)$, using the two retrieval methods for different numbers of unit cells corresponding to $n=(2,4,8,18)$. With increasing

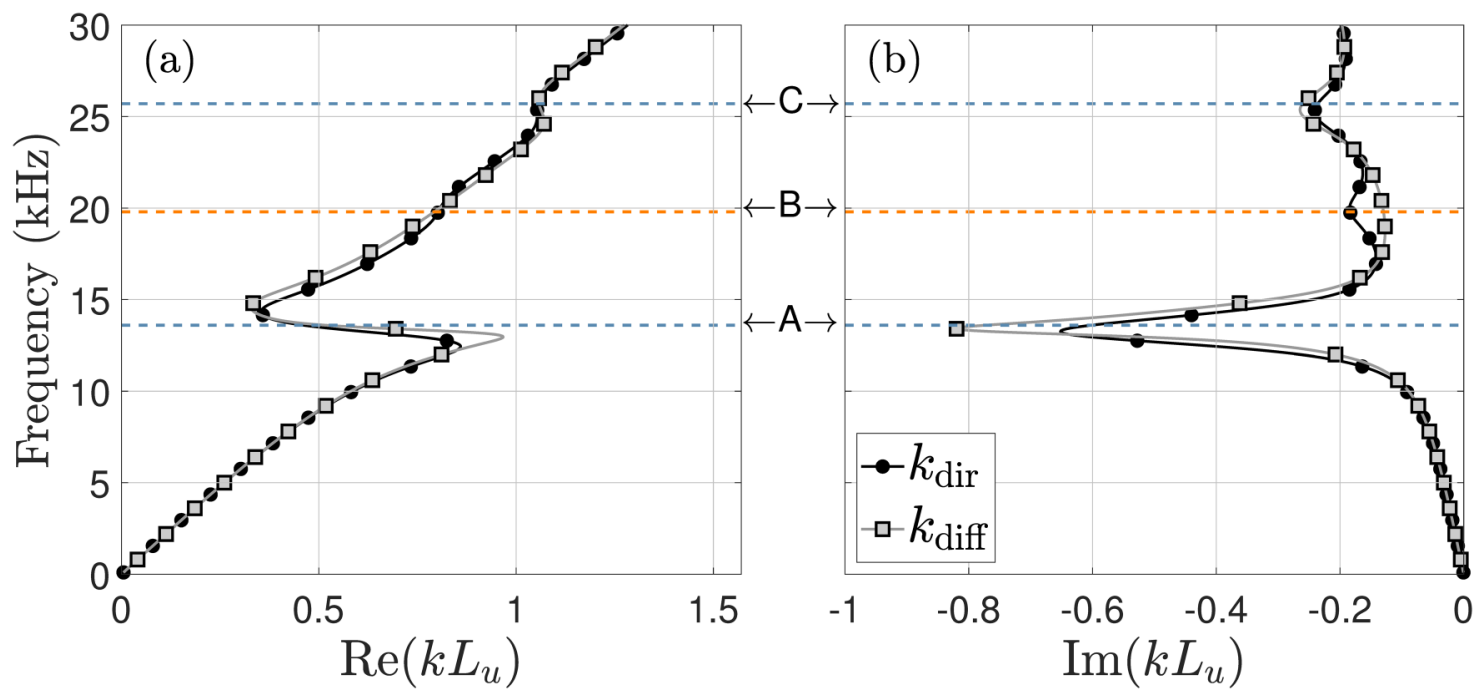

FIG. 6. Dispersion curves for (a) the real part and (b) imaginary part of the reduced effective wavenumber for a locally resonant unit cell obtained with the direct inversion method $\left(k_{\text {dir }}\right)$ and with the differential method $\left(k_{\text {diff }}\right)$. The dashed lines highlight the three selected frequencies A, B, and C. 

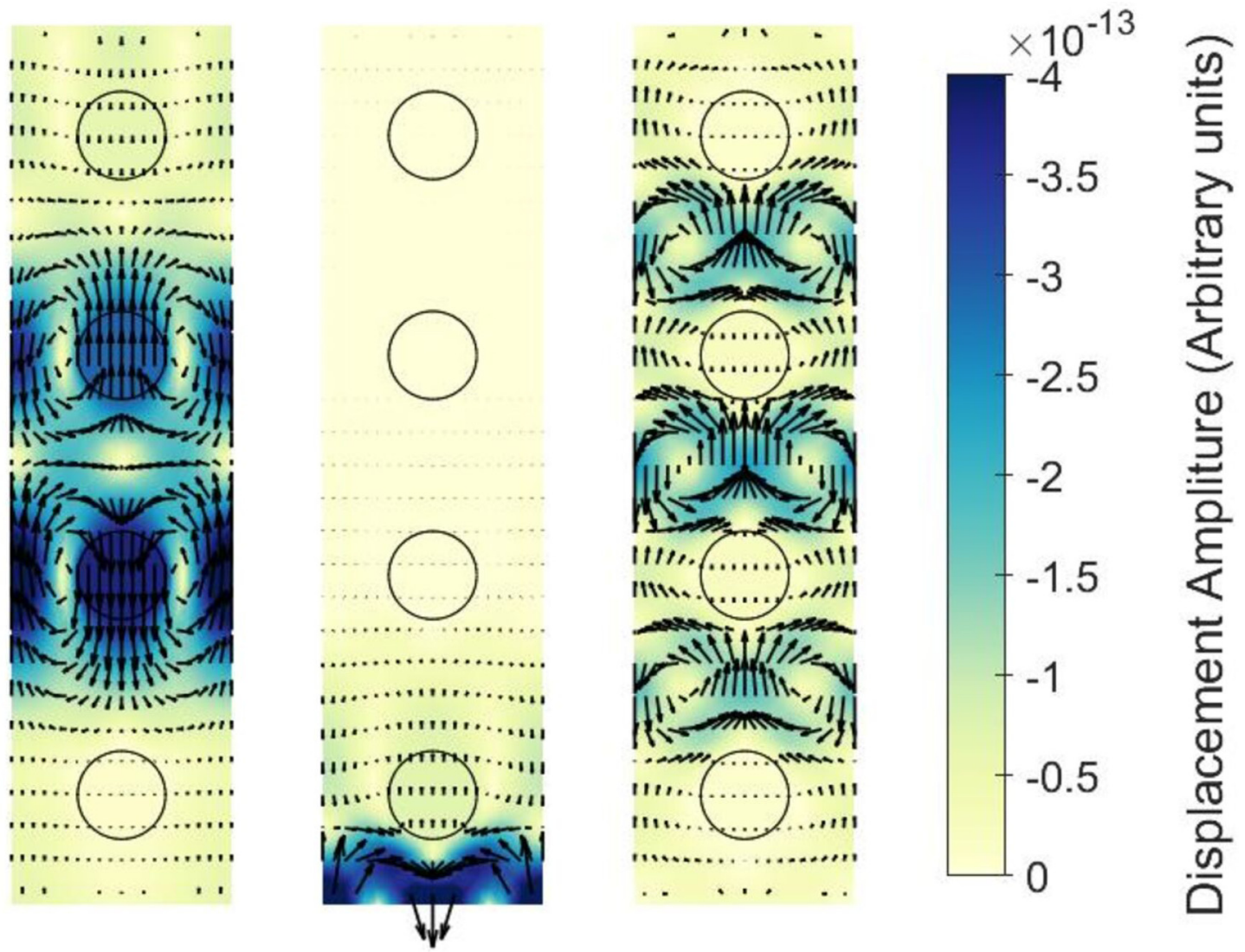

\section{$\mathbf{A}-13.6 \mathrm{kHz} \quad \mathbf{B}-19.8 \mathrm{kHz} \quad \mathbf{C}-25.7 \mathrm{kHz}$}

FIG. 7. Displacement fields for the four-unit medium at given frequencies A, B, and C. The arrows indicate the direction of displacement and their length is proportional to the displacement modulus.

number of unit cells, the effective wavenumber using the direct inversion method converges toward the result obtained using the differential method, corresponding to the effective wavenumber in the medium interior away from boundary interface effects. More specifically, we observe that, at the interface resonance around $20 \mathrm{kHz}$, the direct inversion method is strongly influenced by the number of unit cells $n$, whereas the differential method always accurately removes the interface effects.

Effective properties from both methods are now used to characterize effective media in order to predict the reflection and transmission coefficients of a segmented metamaterial comprising $N$ unit cells. Effective media are thus given the length of $L=N \times L_{u}$. The effective parameters used were obtained for $n=4$ and shown in Figs. 6 and 8 . The scattering coefficients of the effective medium are then compared to coefficients derived from a FE model of the metamaterial comprising $N$ unit cells, as shown in Fig. 10(a). Figures 10(b) and 10(c), respectively, show the corresponding effective medium characterized by the effective wavenumber and effective impedance obtained using the two retrieval methods. A hybrid effective medium is also introduced in order to take advantages of both methods, by using the effective properties of the direct inversion method at the interfaces and the effective properties from the differential method for the interior of the effective medium. The hybrid medium is, therefore, made of three layers as shown in Fig. 10(d).

Results are presented in Fig. 11 for $N=5,9,19$ unit cells, using the effective properties derived with the direct inversion method for $n=4$ and with the differential method with $(n=4$, $n+1=5$ ). All three effective media represented in Figs. 10(b)-10(d) accurately predict the reflection and transmission coefficients up to $15 \mathrm{kHz}$. Above this frequency, the effective parameters from the direct inversion method are strongly dependent on the parameter $n$, particularly for the interface mode according to Fig. 9(a). As such, using the direct inversion method, the scattering coefficients for a high number $N$ tend to present overestimated interface effects since the effective properties have been obtained for a smaller number $n$. For the reflection coefficient, the impedance of the first 

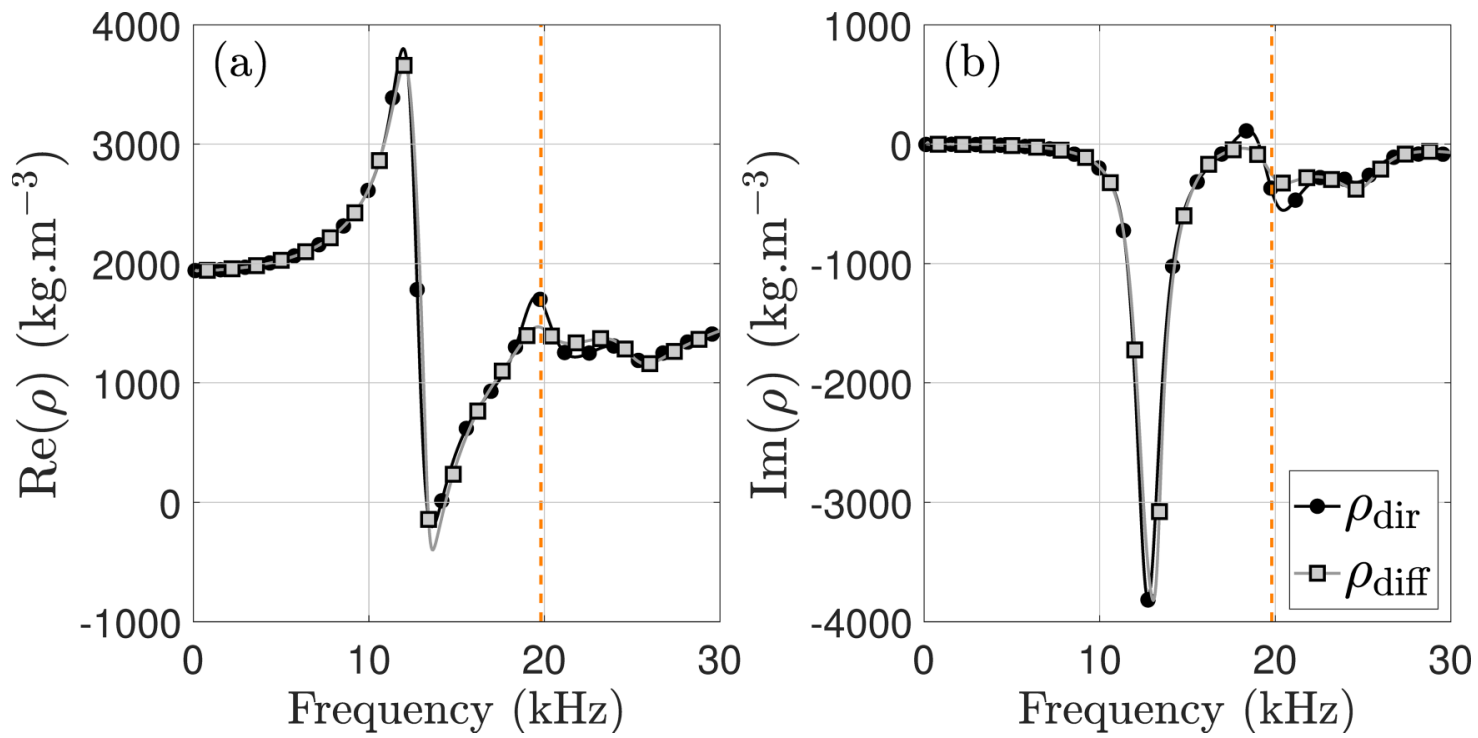

FIG. 8. (a) Real part and (b) imaginary part of the effective density for a locally resonant unit cell obtained with the direct inversion method $\left(\rho_{\text {dir }}\right)$ and with the differential method $\left(\rho_{\text {diff }}\right)$.

layer plays a major part in the results. Both the direct inversion method and the hybrid method are, therefore, less suitable for the prediction of the reflection coefficient for various $N$-layer media than the differential method. For the transmission coefficient, each properties characterizing the medium is relevant. Accurate predictions are obtained using the differential method, except for the transmission dip around $20 \mathrm{kHz}$, that results from the interface mode. Therefore, the transmission coefficient is here better predicted using the hybrid effective medium, as it physically restricts the effective properties of the direct inversion method to the interfaces and the effective properties from the differential method to the core of the effective medium.
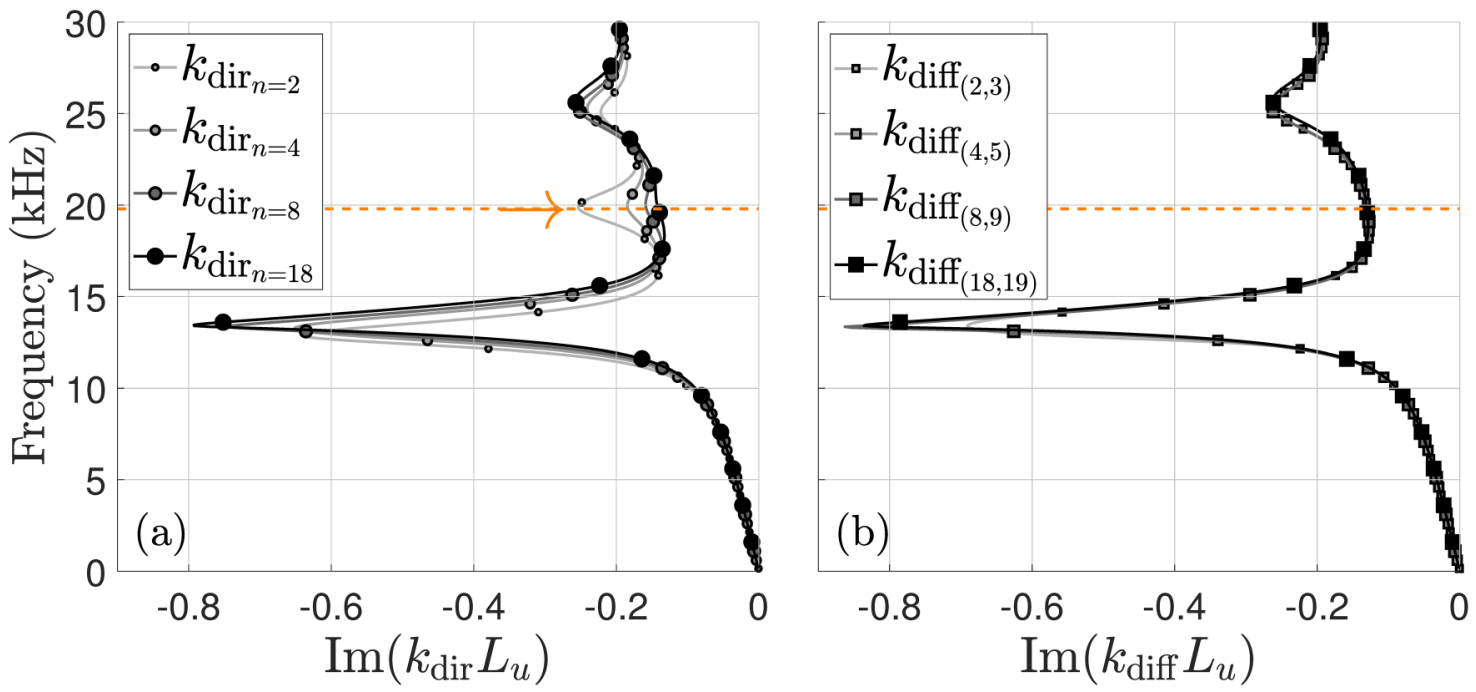

FIG. 9. Imaginary part of the effective wavenumbers obtained using the (a) direct inversion method $k_{\text {dir }_{n}}$ and (b) differential method $k_{\text {diff }}(n, n+1)$ for $n=(2,4,8,18)$, where $n$ is the number of unit cells in the shorter medium. 
(a)
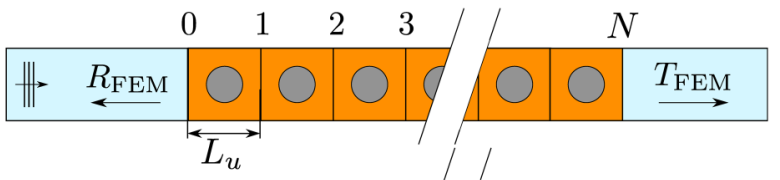

(b)
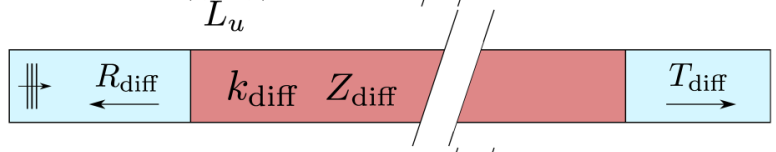

(c)
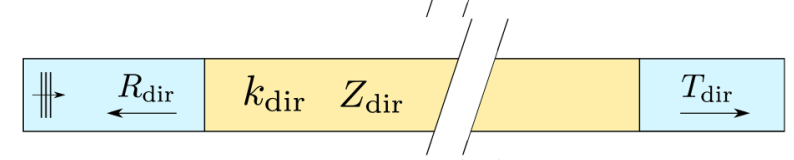

(d)

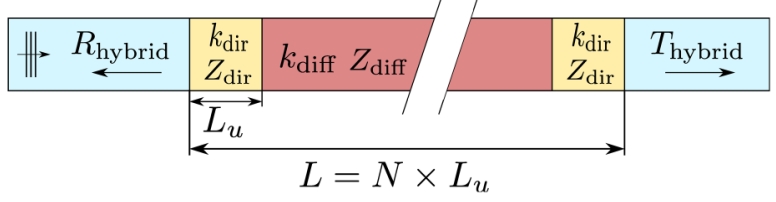

FIG. 10. (a) Finite periodic medium of length comprising $N$ unit cells, for which the reflection and transmission coefficients are predicted using an effective medium characterized by the effective wavenumber and impedance obtained using (b) the differential method (diff), (c) the direct inversion method (dir), (d) a hybrid effective medium comprising the direct inversion method at the boundary interfaces and the differential method for the medium away from the interfaces.
The hybrid effective medium proposed herein is the most intuitive one, with effective properties characterizing edge cells at the input and output edges and effective properties for propagation in the medium interior for inner cells. Moreover, the accuracy is satisfactory for the leading goal mentioned in the introduction. Nevertheless, it is worth noting that other hybrid effective media could be considered, by changing the affectation of effective properties along the medium, for example. However, there is no ideal combination since one working well for a type of system may be less efficient for another type, made of a softer rubber matrix or with another fluid surrounding, for example, for which fluid-structure interactions or near-field effects are different. Predictions could also be improved by considering other models than the fluid homogenization model. The significant effects of the interface resonance observed in the case study imply that a non-local model may better represent the system. In such a model, additional terms could be introduced to model the coupling between a normally incident plane wave and waves that exhibit a transverse wavenumber equal to multiples of the lattice reciprocal vector along the transverse direction. Such waves are involved both in the development of the complex near field at the fluid-structure interface and in the near-field coupling effects between the scatterers within the lattice. The complexity of the homogenization process would then be considerably increased, which would be a major drawback for our leading goal and for the experimental application of the methods.
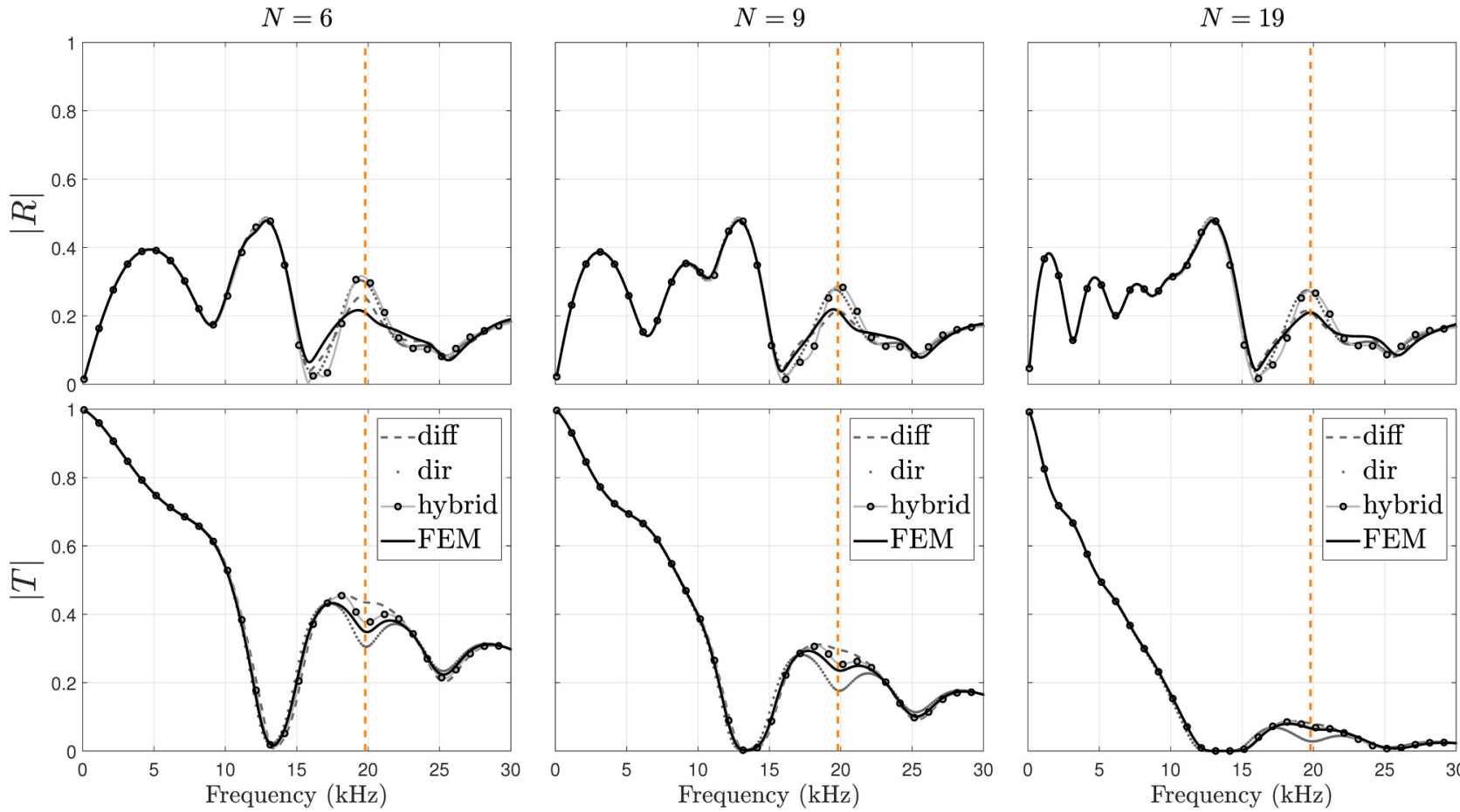

FIG. 11. Reflection and transmission coefficients calculated for the effective media characterized by effective parameters of the differential method (dashed lines) or the direct inversion method (dotted lines) that represent a periodic media comprising $N=5, N=9$, or $N=19$ unit cells. Scattering coefficients from the hybrid effective medium (circle markers) and from FEM (full lines) are also presented. 


\section{CONCLUSION}

Two retrieval methods have been introduced, corresponding to the direct inversion method and the differential method. The direct inversion method uses the reflection and transmission coefficients of a medium comprising a number of identical unit cells, immersed in a fluid, while the differential method requires two segmented media comprising $n$ and $n+1$ unit cells. Both retrieval methods lead to an effective wavenumber and an effective impedance of a homogeneous fluid medium. The effective wavenumber from the differential method was able to describe the interior of the medium, whereas the direct inversion method captures the effects related to the medium's finite size and interfaces with the surrounding fluid media on the incidence and transmission sides. As such, to predict the scattering coefficients, a hybrid effective medium was built, whereby the first and last layers are defined with the effective properties from the direct inversion method and the core layer is defined by the differential method. Reflection and transmission coefficients have been predicted using effective media characterized by the direct inversion method, by the differential method, and by the hybrid medium. The reflection coefficient is accurately predicted by the differential method. The hybrid medium provides accurate predictions of the transmission coefficient as it physically restricts the effective properties of the direct inversion method to the interface and uses the differential method to describe the core of the medium.

The retrieval methods provided here may, therefore, be beneficial to get a better understanding of the wave propagation in a metamaterial coating. Moreover, the hybrid method is an efficient tool to predict and optimize the acoustic performance of coating designs that may comprise less classical inclusions. In a future work, the retrieval methods will be applied to several metamaterial designs in order to create a database of effective properties associated with various inclusion shapes, sizes, and materials. Effective properties will then be used in an optimization process in order to obtain systems with a good acoustic performance over a wide frequency range. The work in the present paper is, therefore, useful to quickly and easily make a topological optimization of acoustic coatings for underwater applications using fluid properties.

In general, the goal of this work is to build homogeneous equivalent media which are a precise approximation of their microstructured counterparts. Effective media can be used to study metamaterials with complex or arbitrary geometries for which analytical models do not apply. The proposed homogenization approach may be especially useful in the study of large metamaterial-based systems including structural gradients, such as the ones considered in transformation acoustics designs. Similarly, the proposed homogenization approach could prove useful in the study of multiscale metamaterials to alleviate the simulation issues related to the presence of complex geometries at different scales, by allowing the replacement of the smaller structures by equivalent media.

\section{ACKNOWLEDGMENTS}

The authors would like to acknowledge Nicole Kessissoglou (UNSW, Sydney) for her assistance with editing. This work was supported by Naval Group Research and a grant from ANRT (Association Nationale de la Recherche et de la Technologie).

\section{DATA AVAILABILITY}

The data that support the findings of this study are available from the corresponding author upon reasonable request.

\section{REFERENCES}

${ }^{1}$ G. Ma and P. Sheng, Sci. Adv. 2, 1501595 (2016).

${ }^{\mathbf{2}}$ N. Kaina, F. Lemoult, M. Fink, and G. Lerosey, Nature 525, 77 (2015).

${ }^{3}$ S. Guenneau, A. Movchan, G. Pétursson, and A. Ramakrishna, New J. Phys. 9, 399 (2017).

${ }^{4}$ N. Boechler, G. Theocharis, and C. Daraio, Nat. Mater. 10, 665 (2011).

${ }^{5}$ H. Chen and C. T. Chan, Appl. Phys. Lett. 91, 183518 (2007).

${ }^{6}$ Z. Liu, X. Zhang, Y. Mao, Y. Y. Zhu, Z. Yang, C. T. Chan, and P. Sheng, Science 289, 1734 (2000).

7. Li and C. T. Chan, Phys. Rev. E 70, 055602 (2004).

${ }^{8}$ Y. Lai, Y. Wu, P. Sheng, and Z.-Q. Zhang, Nat. Mater. 10, 620 (2011).

${ }^{9}$ B. Liang, X. Zou, and J. Cheng, J. Acoust. Soc. Am. 124, 1419 (2008).

${ }^{10}$ H. Huang, C. Sun, and G. Huang, Int. J. Eng. Sci. 47, 610 (2009).

${ }^{11}$ G. S. Sharma, A. Skvortsov, I. MacGillivray, and N. Kessissoglou, J. Sound Vib. 443, 652 (2019).

${ }^{12}$ X. N. Liu, G. K. Hu, G. L. Huang, and C. T. Sun, Appl. Phys. Lett. 98, 251907 (2011).

${ }^{13}$ G. S. Sharma, A. Skvortsov, I. MacGillivray, and N. Kessissoglou, Appl. Acoust. 143, 200 (2019).

${ }^{14}$ S. M. Rytov, Sov. Phys. Acoust 2, 68 (1956).

${ }^{15} \mathrm{~W}$. Xu, T. Yang, and W. Wang, Waves Random Complex Media 23, 258 (2013).

${ }^{\mathbf{1 6}}$ A.-C. Hladky-Hennion and J.-N. Decarpigny, J. Acoust. Soc. Am. 90, 3356 (1991).

${ }^{17}$ G. S. Sharma, A. Skvortsov, I. MacGillivray, and N. Kessissoglou, Wave Motion 70, 101 (2017).

${ }^{18}$ P. Langlet, A.-C. Hladky-Hennion, and J.-N. Decarpigny, J. Acoust. Soc. Am. 98, 2792 (1995).

${ }^{19}$ P. J. Wei and Y. P. Zhao, Mech. Adv. Mater. Struct. 17, 383 (2010).

${ }^{20}$ A. Krushynska, V. Kouznetsova, and M. Geers, J. Mech. Phys. Solids 96, 29 (2016).

${ }^{21}$ B. Bianco and M. Parodi, Alta Freq. 45, 107 (1976).

${ }^{22}$ V. Fokin, M. Ambati, C. Sun, and X. Zhang, Phys. Rev. B 76, 144302 (2007).

${ }^{23}$ R. Zhu, G. L. Huang, and G. K. Hu, J. Vib. Acoust. 134, 031006 (2012).

${ }^{24}$ A. V. Amirkhizi, Mech. Mater. 114, 76 (2017).

${ }^{25}$ C. Croënne, A.-C. Hladky-Hennion, J. Vasseur, and B. Dubus, in Ultrasonics Symposium (IEEE, 2010).

${ }^{26} \mathrm{P}$. Méresse, C. Audoly, C. Croënne, and A.-C. Hladky-Hennion, C. R. Mec. 343, 645 (2015).

${ }^{27}$ C.-L. Ding and X.-P. Zhao, J. Phys. D Appl. Phys. 44, 215402 (2011).

${ }^{28}$ H. Chen, S. Zhai, C. Ding, C. Luo, and X. Zhao, J. Appl. Phys. 118, 094901 (2015).

${ }^{29}$ J. H. Park, H. J. Lee, and Y. Y. Kim, J. Appl. Phys. 119, 034901 (2016).

${ }^{30}$ R.-B. Yang and A. K. Mal, J. Mech. Phys. Solids 42, 1945 (1994).

${ }^{31} \mathrm{M}$. Hinders, B. Rhodes, and T. Fang, J. Sound Vib. 185, 219 (1995).

${ }^{32}$ H. Zhao, Y. Liu, J. Wen, D. Yu, and X. Wen, Phys. Lett. A 367, 224 (2007).

${ }^{33}$ H. Meng, J. Wen, H. Zhao, and X. Wen, J. Sound Vib. 331, 4406 (2012).

${ }^{34}$ M. L. Munjal, Acoustics of Ducts and Mufflers with Application to Exhaust and Ventilation System Design (John Wiley \& Sons, 1987).

${ }^{35}$ D. A. Frickey, IEEE Trans. Microwave Theory Tech. 42, 205 (1994).

${ }^{36}$ In Ref. 35, relations " $S$ to ABCD" are to be considered. ABCD matrix is equivalent to transfer matrix $\mathbf{M}$ as defined in Eq. (1), with one difference concerning the definition of the electrical current (or equivalently the acoustical particle velocity) directions on the input and output sides, which are defined in opposite directions for ABCD but in the same direction for $\mathbf{M}$.

${ }^{37}$ M. B. Muhlestein, C. F. Sieck, P. S. Wilson, and M. R. Haberman, Nat. Commun. 8, 15625 (2017).

${ }^{38}$ ISEN, ATILA Finite Element Software Package for the analysis of $2 D$ \& $3 D$ structures based on smart material. 